\title{
Severe COVID-19 illness in an Indian adolescent with autoimmune polyendocrinopathy syndrome-1
}

\author{
Chirantap Oza ${ }^{1}$, Madhura Karguppikar ${ }^{1}$, Vaman Khadilkar ${ }^{2}$, Anuradha Khadilkar ${ }^{3}$ \\ From ${ }^{1}$ Clinical and Research Fellow, ${ }^{3}$ Deputy Director, Department of Endocrine and Growth Unit, Hirabai Cowasji Jehangir Medical Research \\ Institute, ${ }^{2}$ Counsultant Pediatric Endocrinologist, Department of Endocrine and Growth Unit, Jehangir Hospital, Pune, Maharashtra, India
}

\begin{abstract}
Autoimmune polyglandular syndrome-1 (APS-1)also known as autoimmune polyendocrinopathy candidiasis ectodermal dystrophy is a rare autosomal recessive disorder caused by mutation of AIRE gene on chromosome 21q22.3 with an overall prevalence of $<1: 100,000$. Here, we present a 16-year-old male having clinical history of evolution of symptoms for oral candidiasis, hypoparathyroidism, and adrenal insufficiency (AI). He developed rare endocrine and non-endocrine manifestations such as type-1 diabetes (T1D) and autoimmune hepatitis, respectively. The patient while on hormone replacement therapy along with immunosuppressants developed liver cirrhosis and portal hypertension with esophageal varices and candidiasis. Subsequently, he was admitted for complaints of cough, cold and fever and was confirmed to be affected by SARS-CoV-2 by reverse transcription-polymerase chain reaction method. In his prolonged ICU stay of 26 days, he required oxygen therapy, intravenous glucocorticoids, remdesivir, low molecular weight heparin, and hemodynamic support with inotropes. His medical management with subcutaneous insulin therapy and azathioprine was continued. He was discharged after complete resolution of symptoms and negative tests for SARS-CoV-2 and was advised radiological and clinical follow-up. Reports suggest that risk of severe COVID does not increase in patients with AI or autoimmunity. However, our patient possibly developed severe COVID not only due to AI and autoimmunity but also associated rare manifestations like hyperglycemia due to T1D and cirrhosis. Thus, good glycemic control and well-tolerated modern immunosuppressant therapy may be useful in improving prognosis of severe COVID-19 illness in patients with APS-1.
\end{abstract}

Key words: COVID-19, APS-1, Type-1 Diabetes, Cirrhosis

A utoimmune polyglandular syndrome-1 (APS-1) also known as autoimmune polyendocrinopathy candidiasis ectodermal dystrophy (APECED) is diagnosed by presence of two of the following: (1) adrenocortical failure or evidence of adrenal autoantibodies, (2) hypoparathyroidism, and (3) chronic mucocutaneous candidiasis. The first manifestation to develop is candidiasis in infancy followed by hypoparathyroidism at age of 5-7 years and adrenocortical failure by age of 13 years [1].

Type 1 diabetes (T1D) mellitus is relatively infrequent (4-23\%) in APS-1 depending on genetic predisposition with a peak presentation toward middle age. However, no genetic or immunological basis for T1D in APS-1 has been ascertained. The effect of aromatic L-amino acid decarboxylase specificities and AIRE single nucleotide polymorphism association in the development of T1D in APECED patients remains to be unravelled. The autoimmune process carried out by defective

\section{Access this article online}

Received - 03 November 2021

Initial Review - 18 November 2021

Accepted - 07 December 2021

DOI: $10.32677 /$ ijch.v8i12.3202

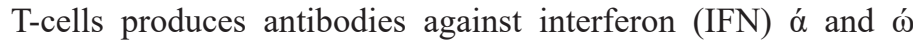
and Th17 cytokines. The role of autoantibodies against IFNs for infectious diseases has recently been suspected, as patients with APS-1 developed severe coronavirus disease 2019 (COVID-19) caused by infection with severe acute respiratory syndrome coronavirus 2 (SARS-CoV-2) [2]. Autoantibodies against IFNs were reported in patients with severe COVID-19. However, to the best of our knowledge there has been no reported case of SARSCoV-2 infection in an Indian patient with APS-1.

\section{CASE PRESENTATION}

We present the case of a 16-year-old boy who is born of $2.75 \mathrm{~kg}$ to non-consanguineous parents with history of hypothyroidism in mother. He first developed oral candidiasis at 2 years of age that required oral anti-candidial treatment for resolution. At the age of 5 years, he developed carpopedal spams, tetany, and hypocalcemic seizures due to hypoparathyroidism. He was started

Correspondence to: Dr. Anuradha Khadilkar, Deputy Director and Consultant Pediatrician, Hirabai Cowasji Jehangir Medical Research Institute, Pune, Maharashtra, India. E-mail: anuradhavkhadilkar@gmail.com

(C) 2021 Creative Commons Attribution-NonCommercial 4.0 International License (CC BY-NC-ND 4.0). 
on calcitriol and calcium supplements and has maintained good compliance since. He was admitted with complaints of diarrhea, fatigue, weight loss, hypotension, and abdominal pain at the age of 10 years and was diagnosed with adrenal insufficiency (AI). Hydrocortisone $\left(15 \mathrm{mg} / \mathrm{m}^{2} /\right.$ day $)$ and fludrocortisone $(50 \mu \mathrm{g})$ were started for the patient for AI and a clinical diagnosis of APS-1 was made.

At the age of 12 years, he developed complaints of polyuria and polydipsia, for which he was evaluated. His blood reports revealed a strongly positive level of glutamic acid decarboxylase (GAD65) antibody (136.2 units/ml), islet cell autoantibodies (ICA-162 units $/ \mathrm{ml}$ ), and c-peptide levels of $0.2 \mathrm{ng} / \mathrm{ml}$. He was confirmed to have T1D and was started on insulin therapy at dose of $1 \mathrm{U} / \mathrm{kg} /$ day by basal bolus regimen. On his baseline evaluation at onset of T1D, the screening for celiac disease (anti-TTG) and hypothyroidism (TSH) was negative. The hemogram was suggestive of pernicious anemia and liver function tests revealed elevated serum glutamic pyruvic transaminase (SGPT) and serum glutamic oxaloacetic transaminase (SGOT) (64 U/L and $67 \mathrm{U} / \mathrm{L})$ with antinuclear antibody positive (1:80) suggestive of autoimmune hepatitis (AIH). The parents gave negative consent for liver biopsy; hence, diagnosis of AIH could not be confirmed.

After 2 years, he developed complaint of blood in vomitus. The abdominal computed tomography (CT) scan was suggestive of splenomegaly, siderotic nodules in splenic parenchyma, liver cirrhosis, dilated portal vein $(1.6 \mathrm{~cm})$ and esophageal varices. An upper gastrointestinal endoscopy was performed (Fig. 1). He was treated with propranolol $60 \mathrm{mg} /$ day in three divided doses and oral fluconazole $150 \mathrm{mg}$ daily. He was also started on treatment with Azathioprine $75 \mathrm{mg} /$ day for AIH.

In our facility, the patient was admitted for complaints of cough, cold and fever and was confirmed to be affected by SARS-CoV-2 by reverse transcription-polymerase chain reaction method. His chest X-ray (Fig. 2) and CT scan were performed. The CT scan was suggestive of non-lobar distribution of ground glass opacity involving bilateral lung parenchyma, crazy paving appearance in bilateral upper lobes, right middle lobe and right lobe with subpleural fibrotic bands. The CT severity score was 23 out of 25 . He required oxygen therapy ( $10 \mathrm{~L} / \mathrm{min})$, intravenous glucocorticoids $\left(15 \mathrm{mg} / \mathrm{m}^{2} /\right.$ day), remdesivir (loading dose $200 \mathrm{mg}$ followed by maintenance dose of $100 \mathrm{mg}$ daily) and low molecular weight heparin at dose of $70 \mathrm{U} / \mathrm{kg} /$ day during his ICU stay. His medical management with subcutaneous insulin therapy and azathioprine was continued. Hemodynamic support required included norepinephrine and dobutamine infusion. During hospitalization, the intravenous glucocorticoid dose was tapered and finally switched back to the pre-admission oral steroid replacement therapy. The patient was discharged after 26 days of hospitalization, with complete resolution of symptoms and negative tests for SARS-CoV-2 and was advised radiological and clinical follow-up.

The patient is currently on therapy with calcitriol $15 \mathrm{ng} / \mathrm{kg} /$ day, alfacalcidiol $25 \mathrm{ng} / \mathrm{kg} /$ day and calcium supplementation at $70 \mathrm{mg} / \mathrm{kg} /$ day for hypoparathyroidism; oral hydrocortisone $10 \mathrm{mg} /$

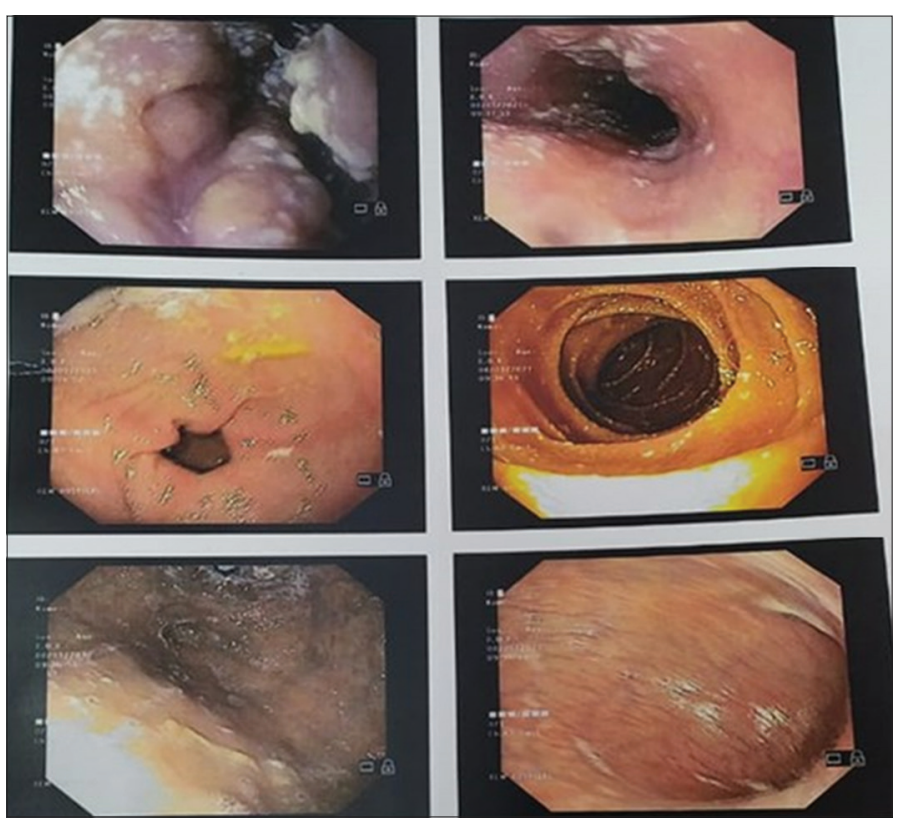

Figure 1: Upper gastrointestinal endoscopy image of the case suggestive of esophageal varices and candidiasis

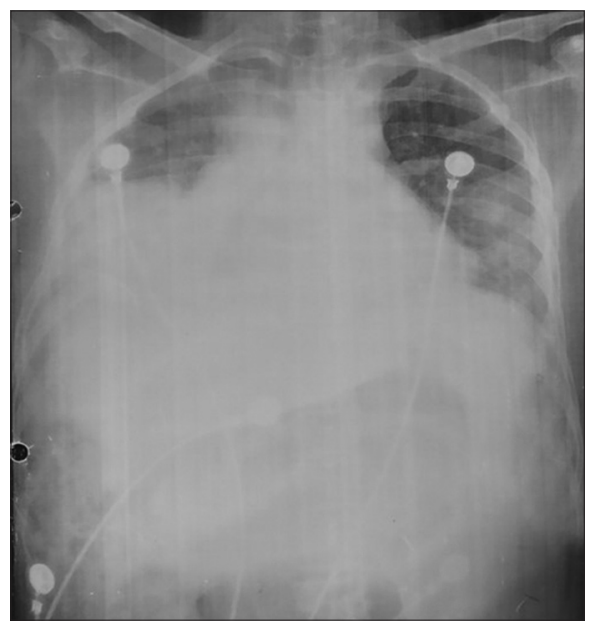

Figure 2: Chest X-ray of the patient at the time of COVID-19 illness

$\mathrm{m}^{2} /$ day and fludrocortisone $50 \mu \mathrm{g} /$ day for $\mathrm{AI}$; insulin therapy at $1.3 \mathrm{U} / \mathrm{kg} /$ day for T1D; azathioprine $75 \mathrm{mg} /$ day for $\mathrm{AIH}$ and propranolol $60 \mathrm{mg} /$ day for varices. At present, his anthropometric measures are height $159 \mathrm{~cm}$ (z-score: -1.1 ), weight $44 \mathrm{~kg}$ (z-score: -1.2 ) and body mass index $17.4 \mathrm{~kg} / \mathrm{m}^{2}$ (z-score: -0.8 ). His $\mathrm{HbAlc}$ is $9.3 \%$, hemoglobin $10.8 \%$, SGPT $37 \mathrm{IU} / \mathrm{L}$, SGOT $53 \mathrm{IU} / \mathrm{L}$, calcium $9.2 \mathrm{mg} / \mathrm{dl}$, phosphorous $3.2 \mathrm{mg} / \mathrm{dl}$, alkaline phosphatase $174 \mathrm{IU} / \mathrm{L}$, vitamin D $35.6 \mathrm{ng} / \mathrm{ml}$, intact parathyroid hormone $13 \mathrm{pg} / \mathrm{ml}$ with normal blood pressure, electrolytes and 17-hydroxy-progesterone levels.

\section{DISCUSSION}

Our patient evolved the three cardinal features of APS- 1 by the age of 10 years as has been noted in $67 \%$ of cases [3]. The median number of disease components in any individual patient throughout their life is 5 , with up to 10 manifestations in some subjects. Besides the cardinal features, our patient developed 
T1D, pernicious anemia and AIH which are noted in frequency of $0-23 \%, 13-49 \%$ and $4-43 \%$ respectively in studies on cohorts of APS-1 in Europe and North America [3].

With regard to Addison disease, the European Society of Endocrinology (ESE) states that there is no evidence that patients with AI are at a higher risk of contracting COVID-19 [4]. Also, a prospective study on outcomes of COVID-19 in patients of APS-1 suggested that infections with SARS-CoV-2 caused mild COVID-19 due to increase in titres of autoantibodies against type-I IFNs in APS-1 patients upon infection with SARS-CoV-2. Type-I IFN-mediated inhibition of SARS-CoV-2 replication is abolished by autoantibodies in patients' plasma in vitro and hence authors concluded that, even if pre-existing autoantibodies against type-I IFNs increase the risk for severe COVID-19, penetrance for severe COVID-19 is not complete [5].

Thus, severe COVID infection in our case cannot just be explained by primary AI as a part of APS- 1 syndrome. The American Association for the Study of Liver Disease has noted that AIH is not a risk factor for development of a severe COVID19 infection but patients with cirrhosis and portal hypertension as a result of AIH are at higher risk of severe illness, prolonged hospitalization and mortality due to COVID-19 [6]. Another study also reports that AIH had higher rates of hospitalization, but no increased risk of ICU admissions or death. An international multicentric study revealed that cirrhosis was the strongest predictor for severe COVID-19 in patients with AIH while maintenance of immunosuppression during COVID-19 was not associated with increased risk of severe disease [7].

The ESE also suggests that chronic hyperglycemia affects the immune function and increases the risk of morbidity and mortality due to COVID-19 infection while the Endocrine Society has suggested that children with T1D and COVID-19 were more likely to develop pneumonia and septic shock and have higher mortality as compared to children with COVID-19 without T1D [8]. Another study concluded that odds of COVID-19 related hospitalization and greater illness severity for patients with T1D are 3-4-fold higher than for patients without diabetes [9]. Poor glycemic control ( $\mathrm{HbA} 1 \mathrm{c}>9 \%)$ is the major determinant of poor outcome in children with T1D which was also noted in our case.

Taken together, here we present a case of APS- 1 in an Indian adolescent with rare manifestations such as AIH and T1D who suffered from severe COVID-19 illness. Reports indicate that AI do not increase the risk of severe COVID-19 infection. However, our case suggests that hyperglycemia due to T1D and cirrhosis due to $\mathrm{AIH}$ in addition to $\mathrm{AI}$ and presence of autoantibodies may have contributed to development of severe COVID-19 illness.

\section{CONCLUSION}

Associated endocrine (T1D) as well as serious non-endocrine (AIH) manifestations in patients with APS-1 may be responsible for development of severe COVID-19 illness in them and hence good glycemic control and well tolerated modern immunosuppressant therapy may be useful in improving prognosis.

\section{AUTHOR'S CONTRIBUTIONS}

All the listed authors - Dr. Chirantap Oza, Dr. Madhura Karguppikar, Dr. Vaman Khadilkar, and Dr. Anuradha Khadilkar played a role in the clinical management, planning, execution, analysis, writing of the manuscript and that they all agree and accept responsibility for the contents of the manuscript submitted to IJCH.

\section{REFERENCES}

1. Husebye ES, Perheentupa J, Rautemaa R, Kämpe O. Clinical manifestations and management of patients with autoimmune polyendocrine syndrome type I. J Intern Med 2009;265:514-29.

2. Bastard P, Rosen LB, Zhang Q, Michailidis E, Hoffmann HH, Zhang Y, et al. Autoantibodies against type I IFNs in patients with life-threatening COVID-19. Science 2020;370:4585.

3. Perheentupa J.APS-I/APECED: The clinical disease and therapy. Endocrinol Metab Clin 2002;31:295-320.

4. Puig-Domingo M, Marazuela M, Giustina A. COVID-19 and endocrine diseases a statement from the European society of endocrinology. Endocrine 2020;68:2-5.

5. Meisel C, Akbil B, Meyer T, Lankes E, Corman VM, Staudacher O, et al. Mild COVID-19 despite autoantibodies to type I IFNs in autoimmunepolyendocrine-syndrome Type 1 (APS-1). J Clin Investig 2021;131:e150867.

6. Available from: http://www.aasldfoundation.org/sites/default/files/2020-10/ COVID19-Flyer-AILD.pdf. [Last accessed on 2021 Sep 06].

7. Efe C, Dhanasekaran R, Lammert C, Ebi B, Higuera-de la Tijera F, Aloman C, et al. Outcome of COVID-19 in patients with autoimmune hepatitis: An international multi-centre study. Hepatology 2021;73:2099-109.

8. Casqueiro J, Casqueiro J, Alves C. Infections in patients with diabetes mellitus: A review of pathogenesis. Indian J Endocrinol Metab 2012;16:S27.

9. Gregory JM, Slaughter JC, Duffus SH, Smith TJ, LeStourgeon LM, Jaser SS, et al. COVID-19 severity is tripled in the diabetes community: A prospective analysis of the pandemic's impact in Type 1 and Type 2 diabetes. Diabetes Care 2021;44:526-32.

Funding: None; Conflicts of Interest: None Stated.

How to cite this article: Oza C, Karguppikar M, Khadilkar V, Khadilkar A. Severe COVID-19 illness in an Indian adolescent with autoimmune polyendocrinopathy syndrome-1. Indian J Child Health. 2021; 8(12):430-432. 